\title{
Autonomy Matters: Experiential and Individual Differences in Chosen and Unchosen Solitary Activities From Three Experience Sampling Studies
}

Social Psychological and

Personality Science

I-I I

(C) The Author(s) 2021

(c) (i)

Article reuse guidelines:

sagepub.com/journals-permissions DOI: I0.1 I77/I94855062II 048066 journals.sagepub.com/home/spP

@SAGE

\author{
Dwight C. K. Tse ${ }^{1,2}{ }^{\mathbb{D}}$, Jennifer C. Lay ${ }^{3}$, and Jeanne Nakamura ${ }^{2}$
}

\begin{abstract}
Solitude - the absence of social interaction — can bring both positive and negative experiences. Drawing on self-determination theory, we conducted three experience sampling studies to investigate quality of experience and dispositions associated with activities varying on two dimensions - chosenness (chosen/unchosen) and social context (solitary/interactive). Participants (total $N=283$ ) completed surveys 6-7 times each day over a 7-day period (total: 8,769 surveys). Multilevel modeling confirmed that participants reported the lowest quality momentary experiences when engaged in unchosen (vs. chosen) solitary activities. Further, individuals who spent more time on unchosen solitary activities reported lower meaning in life and satisfaction with life. Extraversion was positively associated with time spent on chosen interactive activities but negatively with chosen solitary activities. Post hoc analyses revealed that people low (vs. high) in extraversion reported lower productivity only during unchosen interactive activities. Chosen (vs. unchosen) solitary activities seem to have a relatively benign impact on quality of experience and well-being.
\end{abstract}

\section{Keywords}

self-determination theory, ecological momentary assessment, social interaction, solitude

Humans are social animals, but even the most sociable people are unlikely to spend all their waking hours with others. Solitude - the absence of social interaction ${ }^{1}$ - is an inevitable part of life (Burger, 1995; Larson, 1990). Although solitude often has negative connotations due to associations with loneliness and isolation, recent research suggests solitude that is freely chosen can be enjoyable and rewarding (e.g., Lay et al., 2020; Long \& Averill, 2003; Nguyen et al., 2019). Some solitary activities - such as writing, contemplating, relaxing, and enjoying nature - can promote positive feelings, life satisfaction, and personal growth (Long et al., 2003; Wang, 2006). We report findings from three experience sampling method (ESM) studies that potentially reconcile contradictory impressions of solitary experiences. These studies reveal how a sense of autonomy may shape whether solitary activities are benign or harmful for immediate experience and well-being. Contrary to previous research examining autonomy over social context (I want to be alone vs. I have to be alone; e.g., Lay et al., 2020), we focus on autonomy over activities (activities I want to do vs. those I have to do) in solitary and interactive contexts. The findings shed light on the importance of maintaining a sense of autonomy during times of mandatory and inevitable social isolation, for example, under social distancing and shelter-in-place orders during a pandemic.
Empirical studies of daily life experiences suggest solitude is common across the life span. Larson (1990) summarized four ESM studies with samples ranging from schoolchildren to retirees, in which people reported being alone $17 \%-48 \%$ of the time. Later studies using ESM and day reconstruction methods have confirmed solitude's prevalence across the life span (e.g., the oldest-old are alone up to $71 \%$ of the time; Chui et al., 2014) and across cultures (Averill \& Sundararajan, 2014; van Zyl et al., 2018). This suggests that solitude is a universal social condition, and that time spent in solitude varies greatly across individuals.

Solitude is often described with a shade of negativity, as it is often accompanied by loneliness or unmet social needs (Shaver \& Buhrmester, 1983). Because loneliness has deleterious impacts on physical and psychological well-being (Hawkley

\footnotetext{
'University of Strathclyde, Glasgow, UK

${ }^{2}$ Claremont Graduate University, Claremont, CA, USA

${ }^{3}$ The University of Exeter, UK

Corresponding Author:

Dwight C. K. Tse, School of Psychological Sciences and Health, University of Strathclyde, Glasgow GI IQE, UK.

Email: dwight.tse@strath.ac.uk; cheuk-kit-dwight.tse@cgu.edu
} 
$\&$ Cacioppo, 2003), one view is that we must tolerate or overcome solitude when companionship is unavailable. However, the reality is more nuanced. For example, Larson and colleagues (1980) revealed that for adolescents, solitude is no less voluntary than other aspects of daily life. This, along with Pedersen's (1979) work on privacy-seeking and Burger's (1995) studies of individual differences in preference for solitude, suggests people are active agents who voluntarily engage in solitary activities some of the time.

Solitude by its nature enables individuals to engage in activities of their choosing (Long \& Averill, 2003). Earlier work, for example, has emphasized how freedom from social obligations allows individuals to choose their thoughts and actions, an important benefit of solitude (Hammitt, 1982). People derive experiential and well-being benefits from a multitude of activities without any social interaction. These include leisure activities that replenish energy, productive activities that require concentration, creative endeavors, and spiritual/enlightenment pursuits (e.g., Long \& Averill, 2003; Pedersen, 1997, 1999; Wang, 2006).

\section{Positive and Negative Experiences in Solitude}

A growing literature has examined associations between moments spent alone (vs. with others) and various affective states (see Liu et al., 2019, for a recent meta-analysis). Being alone has often been linked with feelings of loneliness (e.g., Hawkley \& Cacioppo, 2003; Pressman et al., 2005) and with low-arousal states (e.g., calm, drowsy; Larson, 1990; Nguyen et al., 2019; Pauly et al., 2017). Although the affective correlates of solitude are multifaceted, if we consider the overall balance between positive and negative affect, time alone is generally linked with greater negative affect than time with others (Larson, 1990; Lay et al., 2019; Liu et al., 2019).

Aside from affective balance, depending on the outcome of interest, solitary activities may not always be less desirable than interactive ones. Due to reduced social obligations and increased privacy, solitary activities are often linked with heightened meaningfulness (e.g., through spiritual activities; Pedersen, 1997, 1999) and stronger engagement and absorption (e.g., Long \& Averill, 2003). With more control over the immediate environment, people are also likely to prefer engaging in creative and productive activities in solitude rather than with others (Larson, 1990; Long et al., 2003). Nonetheless, individuals also report lower vitality and motivation during solitary (vs. interactive) activities (e.g., Larson et al., 1985, 1990; Nguyen et al., 2018). These nuanced results refute the simple dichotomy of solitude "bad" and social interaction as "good". As suggested in previous work (e.g., Chua \& Koestner, 2008), the present study draws on self-determination theory to advance our understanding of "benign" and "harmful" types of solitude.

\section{Autonomy Matters: Chosen and Unchosen Solitary Activities}

According to self-determination theory, autonomy is one of the three basic psychological needs that facilitate optimal human functioning (Ryan \& Deci, 2000), and the satisfaction of these needs is associated with immediate positive feelings and long-term physical and psychological benefits (e.g., $\mathrm{Ng}$ et al., 2012). Momentary felt autonomy has also been linked with better solitary experiences specifically (Lay et al., 2020; Nguyen et al., 2018). As such, meeting the psychological need for autonomy may help determine whether solitude is experienced positively. Autonomy in solitude can be achieved by two means. The first is having one's desired level of social interaction at that moment (autonomy over social context). For example, people can choose to play basketball alone or be forced to play alone if there is nobody around. Previous studies have, however, reported inconsistent findings regarding the impact of autonomy over social context on solitary experiences. A cross-sectional survey study revealed that controlled motivation for solitary activities (e.g., "I'm forced by some external factor to spend time alone"; Chua \& Koestner, 2008, p. 646), which is the opposite of autonomous motivation, exacerbates the negative impact of solitude on well-being. Another study also linked autonomous motivation for solitude with positive outcomes such as greater self-esteem and lower loneliness (Nguyen et al., 2019). Nevertheless, an ESM study (Lay et al., 2020) showed no association between momentary desire for solitude and affective states (when actual social situation was taken into account). Similarly, a longitudinal study (Weinstein \& Nguyen, 2020) found no support for hypothesized associations between overall preference and motivation for solitude and well-being over 2 weeks. Taken together, autonomy over social context (solitude vs. interaction) does not have a consistent effect on affective balance.

Aside from autonomy over social context, another aspect of autonomy concerns whether the activities at hand are volitional or self-selected. In the basketball example, people may choose to practice basketball because they enjoy it (intrinsic motivation) or may practice because of college scholarship requirements (extrinsic motivation) even if they have lost interest in the game. As such, autonomy over one's choice of what activities to do is conceptually distinct from autonomy over one's choice of with whom to do the activities. Moreover, activity choice is more consistent with the notion of "freedom of choice with respect to actions and thoughts," a noted benefit of solitude (Long \& Averill, 2003, p. 24). Although studies outside of the solitude literature have consistently reported salubrious effects of autonomy over activity choice on affect, vigor, and other immediate experiences (e.g., Bachmann et al., 2019; Fullagar \& Kelloway, 2009; Trougakos et al., 2014), little is known about whether chosen activities are experienced more positively in solitary contexts (i.e., "chosen solitary activities"). We conceptualize chosen solitary activities as solitary activities that people want to do and unchosen solitary activities as those that people do not want to do. Extrapolating from existing findings, people should have more positive experiences (affective balance and states such as meaningfulness and engagement) during chosen rather than unchosen solitary activities. 
Beyond immediate experiences, the distinction between chosen and unchosen solitary activities may also clarify individual differences related to solitude and social interaction. Extraversion is a disposition indicative of sociability; however, previous findings are mixed as to whether people high (vs. low) in extraversion spend less time in solitary contexts, whereas research more consistently suggests that people high (vs. low) in extraversion experience less enjoyment in solitary contexts (e.g., Argyle \& Lou, 1990; Pavot et al., 1990; Roozen et al., 2009). Such inconsistent findings may be attributable to previous studies' conflating chosen with unchosen solitary activities. As chosen solitary activities reflect preferences and pleasures derived from such activities, time devoted to them should be negatively associated with extraversion. Unchosen solitary activities, however, should show no such association with extraversion.

Although solitude (vs. interaction) is associated with greater loneliness and poorer psychological well-being (e.g., lower meaning in life; Stillman et al., 2009), advising people to maximize happiness by minimizing overall time spent on solitary activities overlooks the potential benefits of solitude (e.g., Larson, 1997). Considering potential salubrious effects of autonomy on psychological well-being (e.g., Reis et al., 2000; Vansteenkiste \& Ryan, 2013), the negative impacts of solitude on well-being may be negated by having a sense of autonomy over the activities at hand. Conversely, people engaging more in unchosen solitary activities may suffer the "double vulnerability" of a lack of social connectedness and a lack of autonomy, resulting in lower psychological well-being. Overall, psychological well-being should be negatively associated with time spent on unchosen, rather than chosen, solitary activities.

\section{The Current Studies}

Based on self-determination theory and existing research, chosen solitary activities may constitute a more "benign" type of solitude than unchosen solitary activities. In relation to immediate experience, personality, and psychological well-being, we propose the following:

H1: People have better immediate experiences (greater perceived meaningfulness, engagement, and positive affective balance) in chosen than unchosen solitary activities.

H2: People enjoy better cognitive and motivational states (heightened perceived creativity, vitality, productivity, control, and intrinsic motivation) in chosen than unchosen solitary activities.

H3: People high in extraversion spend less time on chosen (but not unchosen) solitary activities.

H4: Spending more time on unchosen (but not chosen) solitary activities is associated with poorer psychological well-being (lower satisfaction with life and meaning in life).
These hypotheses regarding in situ experiences of chosen and unchosen solitary activities have not been investigated previously. Long and colleagues (2003) and Leary and colleagues (2003), for example, examined purposes and types of solitary activities using retrospective reports. Chua and Koestner (2008) and Nguyen and colleagues (2018) used daily diary designs to capture within-person variability in solitary experiences, but reports were retrospective, summarizing overall experiences each day. We instead tested our hypotheses using ESM (Hektner et al., 2007; cf. Larson, 1990; Lay et al., 2020). Participants received signals at random times throughout each day to report on their activities and experiences at that moment. Because of its shorter reporting delay, ESM is superior to one-time surveys and daily diary studies for capturing experiences in situ with minimal recall biases (Mehl \& Conner, 2012).

\section{Method}

\section{Participants and Procedure}

We used data from three ESM studies. Sample sizes were not predetermined because data were retrieved from other projects (see Analysis Plan for post hoc power analyses). Study 1 involved 73 participants recruited by students from a graduate university in Southern California $\left(M_{\text {age }}=31.48, S D=13.49\right.$; $60.0 \%$ female). Study 2 used an online sample of 67 adults recruited through social media $\left(M_{\mathrm{age}}=25.30, S D=7.29\right.$; $71.1 \%$ female). Study 3 involved 203 older volunteers in the United States ( $M_{\text {age }}=71.22, S D=6.13 ; 59.1 \%$ female $)$. Demographics are presented in Table 1. Participants were signaled by a smartphone app at random times six (Studies 1 and 3 ) or seven (Study 2) times a day for seven consecutive days. At each signal, participants answered a short survey about their momentary thoughts, feelings, and behavior. After the 7 days, participants in all studies completed an end-of-week survey.

\section{Measures}

Current activities. Upon being signaled, participants answered questions about the main activity they were doing at that moment. First, they reported why they were doing that activity by selecting one of four options: "I want to," "I want to and I have to," "I have to," and "There is nothing else to do." We coded the first two options as chosen and the last two as unchosen activities. ${ }^{2}$ Participants also reported with whom they were interacting at that moment. We recoded "nobody" as solitary context and other options (e.g., family members, friends) as interactive context. This produced a 2 (activity chosenness: chosen, unchosen $) \times 2$ (social context: solitary, interactive) structure with four categories: chosen solitary activity, unchosen solitary activity, chosen interactive activity, and unchosen interactive activity.

Momentary experiences. We captured momentary meaningfulness, engagement, and affective balance, consistent with Seligman's (2011) components of authentic happiness. At each 
Table I. Descriptive Statistics of Participants in Studies I, 2, and 3.

\begin{tabular}{|c|c|c|c|c|}
\hline \multirow[b]{2}{*}{ Variable } & \multicolumn{4}{|c|}{$M(S D) / \%$} \\
\hline & Study I & Study 2 & Study 3 & Overall \\
\hline$N$ & 66 & 43 & 174 & 283 \\
\hline k & $\mathrm{I}, 874$ & $\mathrm{I}, 244$ & 5,651 & 8,769 \\
\hline Age & 31.48 (13.49) & $25.30(7.29)$ & $71.22(6.13)$ & $55.48(22.22)$ \\
\hline Sex (female) & $60.00 \%$ & $71.05 \%$ & $59.06 \%$ & $60.97 \%$ \\
\hline \multicolumn{5}{|l|}{ Education level } \\
\hline High school or below & $6.67 \%$ & $17.24 \%$ & $2.92 \%$ & $5.38 \%$ \\
\hline Some college & $13.33 \%$ & $31.03 \%$ & $14.62 \%$ & $16.15 \%$ \\
\hline Bachelor's degree & $46.67 \%$ & $31.03 \%$ & $29.24 \%$ & $33.46 \%$ \\
\hline Master's degree & $28.33 \%$ & $10.35 \%$ & $37.43 \%$ & $32.31 \%$ \\
\hline Professional degree & $5.00 \%$ & $10.35 \%$ & $15.79 \%$ & $12.69 \%$ \\
\hline Relationship status (married) & $18.33 \%$ & $6.90 \%$ & $56.47 \%$ & $42.09 \%$ \\
\hline Ethnicity (White) & $66.67 \%$ & $50.00 \%$ & $84.80 \%$ & $76.63 \%$ \\
\hline \multicolumn{5}{|l|}{ Chosenness and social context } \\
\hline Chosen solitary activities & $36.96 \%$ & $44.50 \%$ & $43.11 \%$ & $41.89 \%$ \\
\hline Unchosen solitary activities & $17.28 \%$ & $15.72 \%$ & $6.59 \%$ & $10.47 \%$ \\
\hline Chosen interactive activities & $34.37 \%$ & $31.71 \%$ & $44.73 \%$ & $40.34 \%$ \\
\hline Unchosen interactive activities & II.39\% & $8.07 \%$ & $5.56 \%$ & $7.30 \%$ \\
\hline \multicolumn{5}{|l|}{ Physical location } \\
\hline Home & $44.71 \%$ & $43.25 \%$ & $55.75 \%$ & $51.54 \%$ \\
\hline Work/school & $25.86 \%$ & $26.53 \%$ & $18.33 \%$ & $21.15 \%$ \\
\hline Other & $29.43 \%$ & $30.23 \%$ & $25.92 \%$ & $27.31 \%$ \\
\hline
\end{tabular}

momentary survey, participants in all studies reported the extent to which the activity they were doing was meaningful and the extent to which they were engaged in the activity on a scale from 1 (not at all) to 7 (extremely). To measure affective balance, participants in Study 1 reported their current mood on a scale from 1 (very negative) to 7 (very positive). In Studies 2 and 3, participants reported the extent to which (when signaled) they felt calm, excited, happy, sad, anxious, and frustrated (Study 2); and calm, excited, happy, proud, bored, stressed, and discouraged (Study 3) from 1 (not at all) to 7 (extremely). We adapted items from the Affect Valuation Index to cover different combinations of affective valence and arousal (Tsai et al., 2006). We created an affective balance composite by reverse coding the negative affect items and then averaging scores across all items. The between- and within-person reliability coefficients were .97 and .82 (Study 2) and .98 and .75 (Study 3), respectively (Shrout \& Lane, 2012).

Participants also reported on several cognitive and motivational states similar to those in previous research (Larson et al., 1985). In Study 1, they reported to what extent they felt creative on a scale from 1 (not at all) to 7 (extremely) and rated their energy level from 1 (very exhausted) to 7 (very energetic). The Study 3 momentary surveys also included the item on energy level, and additional items asking participants to what extent they felt productive, felt in control of the situation, and wished to do something else (low intrinsic motivation), from 1 (not at all) to 7 (extremely). These items reflect several potential benefits of solitude suggested in previous research (e.g., Altman, 1975; Long et al., 2003).
Person-level trait and well-being measures. In the end-of-week survey, participants in Studies 1 and 3 completed the extraversion scale of the Big Five Mini-Marker Inventory (Saucier, 1994) on a scale from 1 (extremely inaccurate) to 9 (extremely accurate). Participants in Studies 2 and 3 completed the 10-item Meaning in Life Questionnaire (Steger et al., 2006) that assessed presence and search for meaning from 1 (absolutely untrue) to 7 (absolutely true). Participants in all studies completed the 5-item Satisfaction with Life Scale (Diener et al., 1985) scored from 1 (strongly disagree) to 7 (strongly agree). Cronbach's $\alpha \mathrm{s}$ were .89 (extraversion), .89 (presence of meaning), .91 (search for meaning), and .87 (satisfaction with life).

Covariates. Covariates include age, sex, education (from 1 [high school or less] to 5 [professional/doctoral degree]), ethnicity (White vs. others), and relationship status (married vs. other statuses) at the person level, and physical location (home vs. other places) at the momentary level. The latter two covariates were added based on studies showing solitude was more likely among unmarried people and when at home (Lay et al., 2020; Pauly et al., 2018).

\section{Analysis Plan}

To avoid delayed recall in the momentary reports, we included only responses recorded within 30 min of being signaled (Hektner et al., 2007). We retained only participants who completed at least 15 valid reports over the week ( $>2$ responses/day on average; cf. Enders et al., 2016, for alternative procedures 
for missing data handling such as multilevel multiple imputations). The final sample sizes were 66 (Study 1; compliance rate: 90.4\%), 43 (Study 2; 64.2\%), and 174 (Study 3; 85.7\%). The total numbers of valid responses were 1,874 (Study 1), 1,224 (Study 2), and 5,651 (Study 3).

To account for the nested data structure (momentary assessments nested within participants), we used multilevel modeling with maximum likelihood estimation (Gelman \& Hill, 2006). We regressed quality of experience indicators (e.g., meaningfulness, engagement) on activity chosenness (chosen, unchosen), social context (solitary, interactive), the Activity Chosenness $\times$ Social Context interaction, and covariates. We also conducted post hoc comparisons to examine experiential differences between chosen solitary, unchosen solitary, chosen interactive, and unchosen interactive activities. Sample sizes were sufficient to detect Level-1 effects as small as $d= \pm 0.13$ (Study 1), \pm 0.16 (Study 2), and \pm 0.08 (Study 3 ) with power $=.80$ and $\alpha=.05$ (Dong \& Maynard, 2013; Scherbaum \& Pesner, 2019).

Finally, we performed partial correlation analyses to examine relationships between trait and well-being measures and (person-average) proportions of time spent in each type of activity. Because person-level statistical power is determined by the number of participants instead of the number of responses, the studies had limited power for detecting person-level differences (Snijders, 2005). Hence, we conducted partial correlation analyses using data of all studies combined, controlling for person-level covariates. The combined sample size was sufficiently powered to detect an effect size of $\rho= \pm .20$ with power $=.80$ and $\alpha=.05$ (Faul et al., 2007).

\section{Results}

Analysis code and correlation matrices are publicly available at https://osf.io/bq69d/. Correlation matrices are also in Supplementary Tables S1-S4. Raw data are unavailable due to personally identifiable information collected for other projects.

\section{Descriptive Analyses}

We first examined the distribution of responses across different social contexts and activities. Participants reported engaging in solitary activities in about half of their responses, on average (Study 1: 54.2\%; Study 2: 60.2\%; Study 3: 49.7\%). The average percentage of responses classified as chosen solitary activities and unchosen solitary activities were $37.0 \%$ and $17.3 \%$ (Study 1); $44.5 \%$ and $15.7 \%$ (Study 2); and $43.1 \%$ and $6.6 \%$ (Study 3). Hence, solitary activities were common in people's daily lives, and the majority of solitary activities were chosen rather than unchosen. The intraclass correlations (ICC) of the momentary-level quality of experience variables ranged from .186 to .399 (see Table 2), reinforcing the necessity of multilevel modeling (Gelman \& Hill, 2006).

\section{Momentary-Level Analyses: Quality of Experience During Activities}

We used multilevel models (Level 1: momentary-level, Level 2: person-level) to examine effects of activity chosenness, social context, and their interaction on quality of experience (activity meaningfulness, activity engagement, affective balance, creativity, energy, productivity, control, and intrinsic motivation), controlling for covariates. Table 2 and Supplementary Tables S5-S12 report model estimates for each study separately and the three samples combined (with study membership included as a covariate). Across all studies, the positive main effects of activity chosenness on quality of experience were significant (except for creativity), suggesting that quality of experience was consistently higher in chosen than in unchosen activities. Main effects of social context on meaningfulness, engagement, affective balance, energy, productivity, and intrinsic motivation (reverse of "wishing to do something else") were consistently negative across studies, suggesting overall higher quality of experience in interactive than in solitary activities. As expected, however, participants reported feeling more in control in solitary than in interactive activities. Many of the Social Context $\times$ Activity Chosenness interactions were statistically significant; hence, we conducted post hoc comparisons of quality of experience across chosen solitary, unchosen solitary, chosen interactive, and unchosen interactive activities.

Figure 1 reports estimates and $95 \%$ CIs for the outcome variables in each social context and activity chosenness condition. Across studies, participants reported the highest levels of meaningfulness, engagement, and affective balance during chosen interactive activities, followed by chosen solitary, unchosen interactive, and unchosen solitary activities. In Study 1, participants also reported the highest energy levels during chosen interactive activities, although creativity did not differ across activity types. In Study 3, although participants reported the highest levels of energy, productivity, and intrinsic motivation during chosen interactive activities, they reported the greatest sense of control during chosen solitary activities. Taken together, findings supported $\mathrm{H} 1$ and $\mathrm{H} 2$, such that quality of experience in chosen solitary activities was overall better than unchosen solitary activities. The inclusion of chosen/unchosen solitary/interactive activities in models reduced momentary-level variance in the quality of experience outcomes by amounts ranging from $1.96 \%$ (creativity) to 16.93\% (motivation; see Table 2).

\section{Person-Level Analyses: Individual Difference Correlates of Activity Engagement}

We also examined correlations between person-level proportions of time spent in each activity category (chosen/unchosen, social/interactive) and extraversion, meaning in life, and satisfaction with life (see Supplementary Table S4). Extraversion was negatively associated with the proportion of time spent in chosen solitary activities $(r=-.234,95 \%$ CI $[-.352,-.108])$ 
Table 2. Unstandardized Estimates and 95\% Confidence Intervals for Multilevel Models Predicting Quality of Experience in Chosen Solitary Activities, Unchosen Solitary Activities, Chosen Interactive Activities, and Unchosen Interactive Activities in Studies I, 2 , and 3.

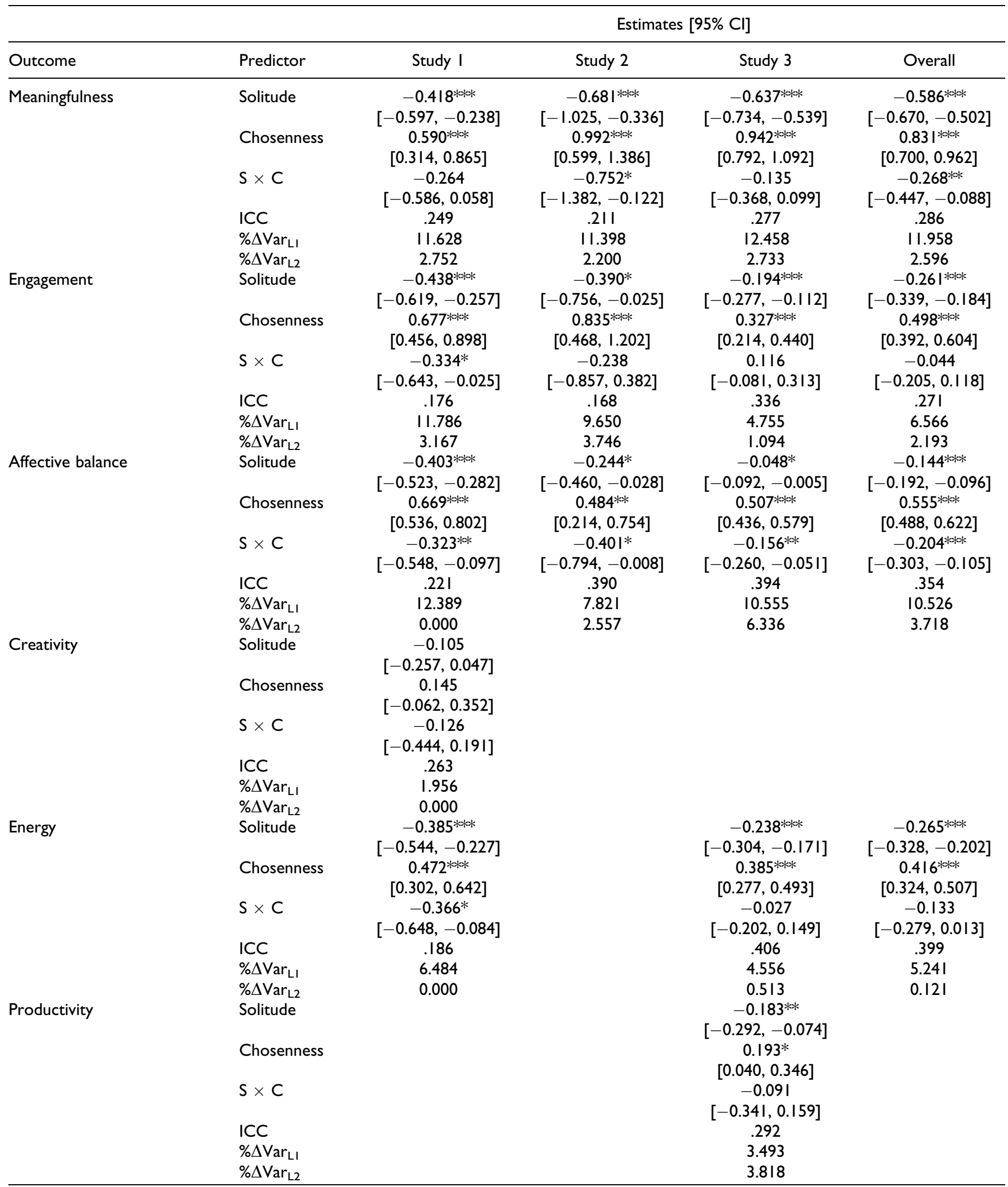


Table 2. (continued)

\begin{tabular}{|c|c|c|c|c|c|}
\hline \multirow[b]{2}{*}{ Outcome } & \multirow[b]{2}{*}{ Predictor } & \multicolumn{4}{|c|}{ Estimates $[95 \% \mathrm{Cl}]$} \\
\hline & & Study I & Study 2 & Study 3 & Overall \\
\hline \multirow[t]{6}{*}{ Sense of control } & Solitude & & & $\begin{array}{c}0.232 * * * \\
{[0.146,0.317]}\end{array}$ & \\
\hline & Chosenness & & & $\begin{array}{c}0.708 * * * \\
{[0.580,0.836]}\end{array}$ & \\
\hline & $\mathrm{S} \times \mathrm{C}$ & & & $\begin{array}{c}-0.223 * \\
{[-0.430,-0.016]}\end{array}$ & \\
\hline & ICC & & & .340 & \\
\hline & $\% \Delta \operatorname{Var}_{\mathrm{LI}}$ & & & 6.199 & \\
\hline & $\% \Delta \operatorname{Var}_{\mathrm{L} 2}$ & & & 4.995 & \\
\hline \multirow[t]{6}{*}{ Wish to do something else } & Solitude & & & $\begin{array}{c}0.212^{* * * *} \\
{[0.113,0.31 \mathrm{I}]}\end{array}$ & \\
\hline & Chosenness & & & $-1.798 * * *$ & \\
\hline & $\mathrm{S} \times \mathrm{C}$ & & & $\begin{array}{c}{[-2.026,-\mid .57 I]} \\
0.327^{*} \\
{[0.058,0.596]}\end{array}$ & \\
\hline & ICC & & & .263 & \\
\hline & $\% \Delta \operatorname{Var}_{\mathrm{LI}}$ & & & 16.933 & \\
\hline & $\% \Delta \operatorname{Var}_{\mathrm{L} 2}$ & & & 13.905 & \\
\hline
\end{tabular}

Note. For the three studies combined, $N=283 ; k=8,749$. For Study I, $N=66 ; k=1,874$. For Study $2, N=43 ; k=1,224$. For Study $3, N=174 ; k=5,65 I$. $\mathrm{S} \times \mathrm{C}=$ Two-way interaction between social context (solitary vs. interactive) and activity chosenness (chosen vs. unchosen). ICC $=$ Intraclass correlation. $\% \Delta \operatorname{Var}_{\mathrm{LI}}$ and $\% \Delta \operatorname{Var}_{\mathrm{L} 2}$ are the percentages of Level-I (momentary-level) and Level-2 (person-level) variances reduced by including social context, activity chosenness, and their interaction in models containing only the covariates. Study 3 was the comparison group for the overall analyses. For parsimony, estimates of covariates, random effects, and intercepts are reported in Supplementary Tables S5-SI2.

$*_{p}<.05 . * * p<.01$. *** $p<.001$.

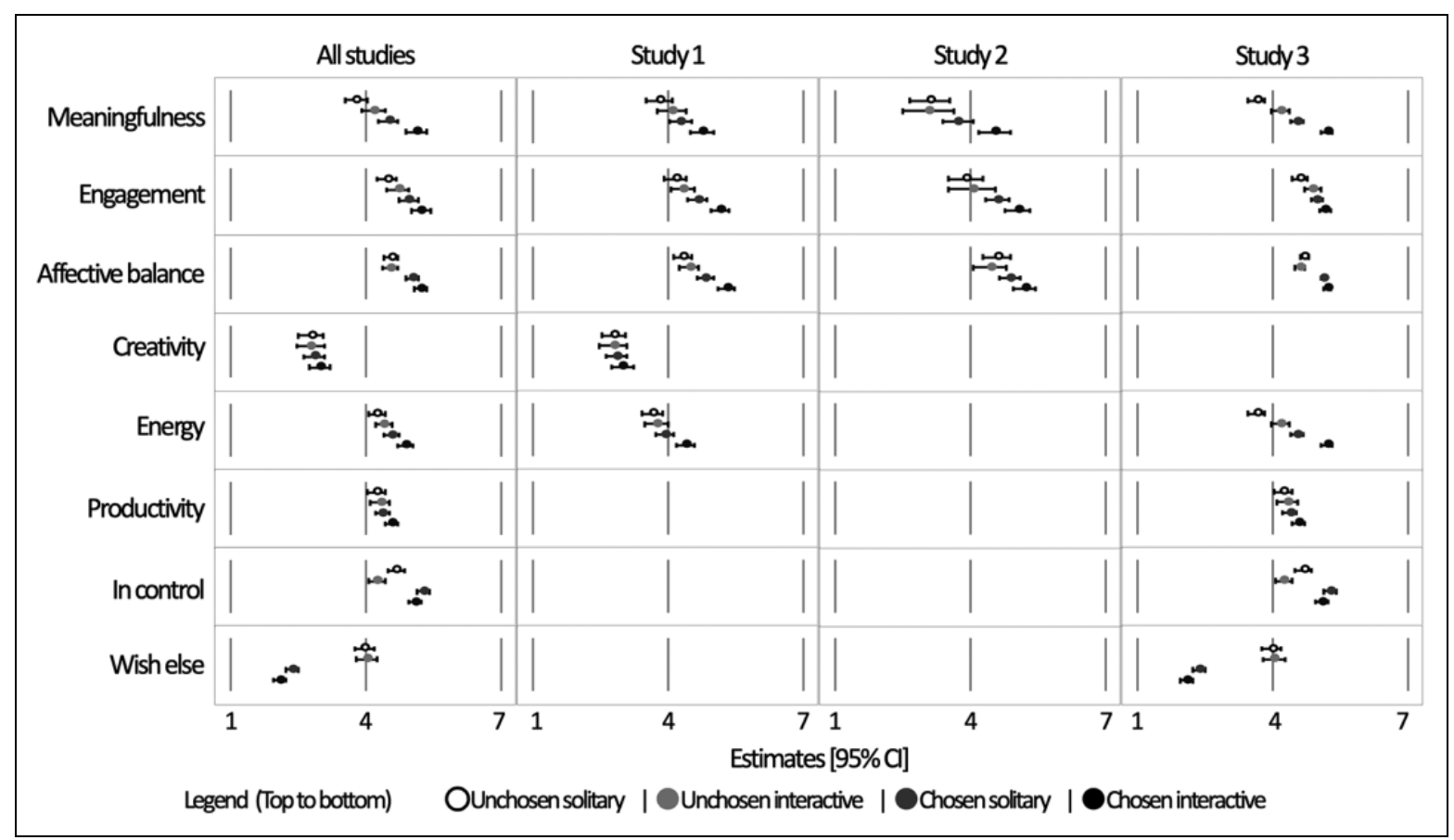

Figure I. Unstandardized estimates and $95 \%$ confidence intervals for quality of experience in chosen/unchosen solitary/interactive activities across all studies and in Studies I, 2, and 3 (from left to right). Note. For the three studies combined, $N=283 ; k=8,749$. For Study I, $N=66$; $k=1,874$. For Study $2, N=43 ; k=1,224$. For Study $3, N=174 ; k=5,65 \mathrm{I}$. Wish Else = wish to do something else. 


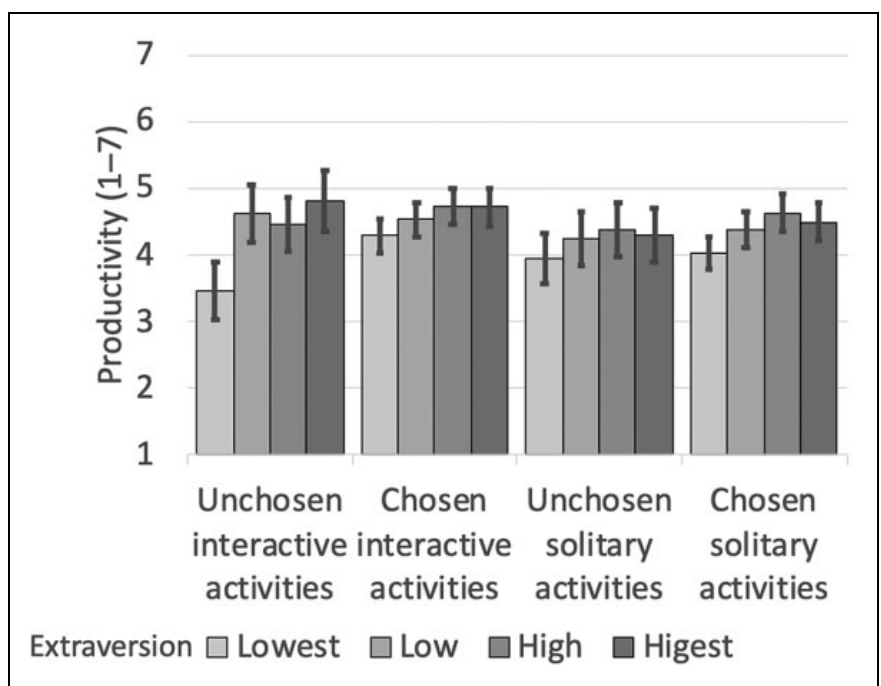

Figure 2. Unstandardized estimates and $95 \%$ confidence intervals of productivity in chosen/unchosen solitary/interactive activities, split by extraversion. Note. $N=174 ; k=5,65$ I. Extraversion was split into quartiles. Estimates were computed adjusted for physical location, age, gender, education, ethnicity, and marital status.

but positively associated with the proportion of time in chosen interactive activities $(r=.205$, CI $[.078, .328])$. That is, people higher in extraversion engaged more in chosen interactive activities and less in chosen solitary activities, consistent with H3. Further, the proportion of time spent in unchosen solitary activities was negatively associated with both presence of meaning in life $(r=-.326$, CI $[-.446,-.195])$ and satisfaction with life $(r=-.275$, CI $[-.384,-.159])$. That is, the more people engaged in unchosen solitary activities, the poorer their psychological well-being, supporting H4. Search for meaning in life showed no associations with engagement in chosen $(r=-.070$, CI $[-.208, .071])$ or unchosen solitary activities $(r=.017$, CI $[-.123, .157])$.

Finally, we conducted post hoc cross-level analyses examining moderating effects of extraversion on quality of experience in chosen/unchosen solitary/interactive activities (see Supplementary Tables S13-S20). The only statistically significant three-way Extraversion $\times$ Solitude $\times$ Chosenness interaction was when predicting productivity $(b=0.307,95 \%$ CI $[0.077$, 0.538]). As shown in Figure 2, less extraverted participants reported lower productivity levels in unchosen interactive activities than their more extraverted counterparts.

\section{Discussion}

We examined people's momentary quality of experience as a function of social context (solitary/interactive) and activity chosenness (chosen/unchosen) across three ESM studies. We also examined whether overall time spent on these different activities was associated with extraversion and psychological well-being variables. Consistent with hypotheses, people reported better quality experiences in chosen than in unchosen solitary activities $(\mathrm{H} 1, \mathrm{H} 2)$. Whereas extraversion was positively associated with time spent on chosen interactive activities and negatively associated with chosen solitary activities (H3), satisfaction with life and presence of meaning in life were negatively associated only with unchosen solitary activities (H4). Post hoc analyses revealed that people low (vs. high) in extraversion felt less productive in chosen interactive activities; however, productivity levels across different levels of extraversion were similar in chosen and unchosen solitary activities (Figure 2). In summary, chosen solitary activities appear to be a relatively "benign" type of solitude, whereas unchosen solitary activities appear relatively "harmful" given their relationships with quality of experience and well-being.

Our findings underscore the importance of felt autonomy in both solitary and interactive contexts. Previous studies have focused on autonomy over the choice between interacting and not interacting with others (Lay et al., 2020; Nguyen et al., 2018). Our studies, however, focus on autonomy over the choice of doing a particular activity (regardless of whether a person wants to do it alone or with others), which may help explain diverging findings in the solitude literature. Future research can disentangle activity choice from choice of social context by measuring these together.

Extending our understanding of autonomous solitude to autonomy over solitary activities is particularly important given the contemporary lifestyles. For example, in the COVID-19 pandemic, social distancing and shelter-in-place orders increase people's risk of social isolation (Krendl \& Perry, 2021). From a conventional perspective focusing on autonomy over social context, people being forced into unwanted solitude may suffer poorer psychological health. However, even when people must stay physically isolated, they can preserve autonomy by doing chosen (rather than unchosen) solitary activities. That is, the psychological impacts of public health measures such as lockdowns may be ameliorated if people spend their self-isolation time doing chosen solitary activities.

\section{Strengths, Limitations, and Future Directions}

Unlike cross-sectional, one-time surveys, ESM studies have the advantage of capturing ecologically valid within-person variation (Hektner et al., 2007). However, ESM studies are costly to conduct, particularly with demographic groups requiring more assistance with ESM smartphone applications. This limits ESM study sample sizes, raising concerns about statistical power and unstable model estimates. We mitigated this sample size issue (especially for between-person analyses) by combining data from three studies, while also reporting results for each study. Another limitation was our use of nonrepresentative samples. For example, older adults able to participate in our smartphone-based ESM study (Study 3) were likely healthier and more educated than their same-age peers. Moreover, individuals who do more solitary activities due to mobility or health limitations were likely underrepresented in our samples. Hence, findings may not be generalizable to population representative samples. 
Due to the time-intensive ESM design, we used single-item measures when possible to reduce respondents' fatigue (Mehl \& Conner, 2012). While this practice limits internal consistency, avoiding lengthy scales is common in ESM studies (e.g., Hektner et al., 2007; Larson, 1990). We also combined positive and negative affect items in Studies 2 and 3 to create the affective balance composite. Findings should be interpreted with caution given the debate over the unidimensionality of positive and negative affect (Diener \& Emmons, 1984; Watson et al., 1988) and the differing affect items used across studies. For most experiential outcomes, chosen and unchosen solitary activities showed similar positive effects in our studies. Future research should replicate these preliminary findings using more established scales (e.g., for creativity, productivity).

Future studies should also expand this work to other personality characteristics and samples. For example, besides extraversion, relationships between chosen/unchosen solitary activities and other personality traits such as neuroticism are worth examining, given previous research linking these traits with solitude preferences (e.g., Burger, 1995). Additionally, our participants were from Western cultures, which value autonomy more than non-Western cultures (Iyengar \& Lepper, 1999). Given the cultural differences in solitary experiences (e.g., Jiang et al., 2019) and autonomy-seeking (e.g., Markus, 2016), cross-cultural studies may reveal different well-being implications of these activity contexts across cultures.

\section{Conclusion}

Going beyond autonomy over social contexts, our three ESM studies examined autonomy over choosing to do specific activities. We show that unchosen (vs. chosen) solitary activities are accompanied by worse quality of experience, and consistent engagement in these activities is associated with poorer psychological well-being. Chosen solitary activities, in contrast, have a relatively benign impact on quality of experience and well-being, and prolonged engagement in these activities is associated with introversion. When people are put into solitude, such as during a lockdown, finding activities they want to do may be key to alleviating negative experiences.

\section{Declaration of Conflicting Interests}

The author(s) declared no potential conflicts of interest with respect to the research, authorship, and/or publication of this article.

\section{Funding}

The author(s) received no financial support for the research, authorship, and/or publication of this article.

\section{ORCID iD}

Dwight C. K. Tse (D) https://orcid.org/0000-0003-2725-1849

\section{Supplemental Material}

The supplemental material is available in the online version of the article.

\section{Notes}

1. Previous research has often defined solitude as the absence of other people in the same space (cf. Larson, 1990, Nguyen et al., 2018). While absence of others usually implies absence of social interaction, these do not always go together. For conceptual clarity, we define solitude as the absence of social interaction. For example, joining a video conference at home would be an interactive activity, whereas studying quietly in a public library would be a solitary activity.

2. The chosen versus unchosen activity distinction emphasizes different motivations for doing an activity. Take reading a novel as an example: We classify this as a chosen activity if a student reads the novel because she finds it interesting, but as an unchosen activity if the student reads the novel just to meet course requirements. These definitions of chosen/unchosen activities are close to Ryan and Deci's (2000) ideas of intrinsic/extrinsic motivation.

\section{References}

Altman, I. (1975). The environment and social behavior: Privacy, personal space, territory, crowding. Brooks/Cole.

Argyle, M., \& Lu, L. (1990). The happiness of extraverts. Personality and Individual Differences, 11(10), 1011-1017. https://doi.org/10. 1016/0191-8869(90)90128-e

Averill, J. R., \& Sundararajan, L. (2014). Experiences of solitude: Issues of assessment, theory, and culture. In R. J. Coplan \& J. C. Bowker (Eds.), The handbook of solitude: Psychological perspectives on social isolation, social withdrawal, and being alone (pp. 90-108). Wiley Blackwell.

Bachmann, O., Grunschel, C., \& Fries, S. (2019). Multitasking and feeling good? Autonomy of additional activities predicts affect. Journal of Happiness Studies, 20(3), 899-918. https://doi.org/10. 1007/s10902-018-9973-3

Burger, J. M. (1995). Individual differences in preference for solitude. Journal of Research in Personality, 29(1), 85-108. https://doi.org/ 10.1006/jrpe.1995.1005

Chua, S. N., \& Koestner, R. (2008). A self-determination theory perspective on the role of autonomy in solitary behavior. The Journal of Social Psychology, 148(5), 645-648. https://doi.org/10.3200/ socp.148.5.645-648

Chui, H., Hoppmann, C. A., Gerstorf, D., Walker, R., \& Luszcz, M. A. (2014). Social partners and momentary affect in the oldest-old: The presence of others benefits affect depending on who we are and who we are with. Developmental Psychology, 50(3), 728-740. https://doi.org/10.1037/a0033896

Diener, E., \& Emmons, R. A. (1984). The independence of positive and negative affect. Journal of Personality and Social Psychology, 47(5), 1105-1117. https://doi.org/10.1037/0022-3514.47.5.1105

Diener, E., Emmons, R. A., Larsen, R. J., \& Griffin, S. (1985). The satisfaction with life scale. Journal of Personality Assessment, 49(1), 71-75. https://doi.org/10.1207/s15327752jpa4901_13 
Dong, N., \& Maynard, R. A. (2013). PowerUp!: A tool for calculating minimum detectable effect sizes and minimum required sample sizes for experimental and quasi-experimental design studies. Journal of Research on Educational Effectiveness, 6(1), 24-67. https://10.1080/19345747.2012.673143

Enders, C. K., Mistler, S. A., \& Keller, B. T. (2016). Multilevel multiple imputation: A review and evaluation of joint modeling and chained equations imputation. Psychological Methods, 21(2), 222-240. https://doi.org/10.1037/met0000063

Faul, F., Erdfelder, E., Lang, A. G., \& Buchner, A. (2007). G*Power 3: A flexible statistical power analysis program for the social, behavioral, and biomedical sciences. Behavior Research Methods, 39(2), 175-191. https://doi.org/10.3758/Bf03193146

Fullagar, C. J., \& Kelloway, E. K. (2009). Flow at work: An experience sampling approach. Journal of Occupational and Organizational Psychology, 82(3), 595-615. https://doi.org/10.1348/0963 $17908 \times 357903$

Gelman, A., \& Hill, J. (2006). Data analysis using regression and multilevel/hierarchical models. Cambridge University Press.

Hammitt, W. E. (1982). Cognitive dimensions of wilderness solitude. Environment and Behavior, 14(4), 478-493. https://doi.org/10. 1177/0013916582144005

Hawkley, L. C., \& Cacioppo, J. T. (2003). Loneliness and pathways to disease. Brain, Behavior, and Immunity, 17(1), 98-105. https://doi. org/10.1016/s0889-1591(02)00073-9

Hektner, J. M., Schmidt, J. A., \& Csikszentmihalyi, M. (2007). Experience sampling method: Measuring the quality of everyday life. SAGE.

Iyengar, S. S., \& Lepper, M. R. (1999). Rethinking the value of choice: A cultural perspective on intrinsic motivation. Journal of Personality and Social Psychology, 76(3), 349-366. http://doi. org/10.1037/0022-3514.76.3.349

Jiang, D., Fung, H. H., Lay, J. C., Ashe, M. C., Graf, P., \& Hoppmann, C. A. (2019). Everyday solitude and well-being in old age: The role of culture versus immigration. Aging and Mental Health, 23(9), 1095-1104. https://doi.org/10.1080/13607863.2018.1479836

Krendl, A. C., \& Perry, B. L. (2021). The impact of sheltering in place during the COVID-19 pandemic on older adults' social and mental well-being. The Journals of Gerontology, Series B: Psychological Sciences, 76(2), e53-e58. https://doi.org/10.1093/ geronb/gbaa110

Larson, R. W. (1990). The solitary side of life: An examination of the time people spend alone from childhood to old age. Developmental Review, 10(2), 155-183. https://doi.org/10.1016/0273-2297(90) 90008-r

Larson, R. W. (1997). The emergence of solitude as a constructive domain of experience in early adolescence. Child Development, 68(1), 80-93. https://doi.org/10.2307/1131927

Larson, R., Csikszentmihalyi, M., \& Graef, R. (1980). Mood variability and the psychosocial adjustment of adolescents. Journal of Youth and Adolescence, 9(6), 469-490. https://doi.org/10.1007/ bf02089885

Larson, R., Zuzanek, J., \& Mannell, R. (1985). Being alone versus being with people: Disengagement in the daily experience of older adults. Journal of Gerontology, 40(3), 375-381. https://doi.org/10. 1093/geronj/40.3.375
Lay, J. C., Fung, H. H., Jiang, D., Lau, C. H., Mahmood, A., Graf, P., \& Hoppmann, C. A. (2019). Solitude in context: On the role of culture, immigration, and acculturation in the experience of time to oneself. International Journal of Psychology, 55(4), 562-571. https://doi.org/10.1002/ijop.12641

Lay, J. C., Pauly, T., Graf, P., Mahmood, A., \& Hoppmann, C. A. (2020). Choosing solitude: Age differences in situational and affective correlates of solitude-seeking in midlife and older adulthood. The Journals of Gerontology Series B: Psychological Sciences, 59(6), 294-304. https://doi.org/10.1093/geronb/gby044

Leary, M. R., Herbst, K. C., \& McCrary, F. (2003). Finding pleasure in solitary activities: Desire for aloneness or disinterest in social contact? Personality and Individual Differences, 35(1), 59-68. https:// doi.org/10.1016/s0191-8869(02)00141-1

Liu, H., Xie, Q. W., \& Lou, V. W. Q. (2019). Everyday social interactions and intra-individual variability in affect: A systematic review and meta-analysis of ecological momentary assessment studies. Motivation and Emotion, 43(2), 339-353. https://doi.org/ 10.1007/s11031-018-9735-x

Long, C. R., \& Averill, J. R. (2003). Solitude: An exploration of benefits of being alone. Journal for the Theory of Social Behaviour, 33(1), 21-44. https://doi.org/10.1111/1468-5914.00204

Long, C. R., Seburn, M., Averill, J. R., \& More, T. A. (2003). Solitude experiences: Varieties, settings, and individual differences. Personality and Social Psychology Bulletin, 29(5), 578-583. https://doi.org/10.1177/0146167203029005003

Markus, H. R. (2016). What moves people to action? Culture and motivation. Current Opinion in Psychology, 8, 161-166. https:// doi.org/10.1016/j.copsyc.2015.10.028

Mehl, M. R., \& Conner, T. S. (Eds.). (2012). Handbook of research methods for studying daily life. Guilford Press.

Ng, J. Y. Y., Ntoumanis, N., Thøgersen-Ntoumani, C., Deci, E. L., Ryan, R. M., Duda, J. L., \& Williams, G. C. (2012). Selfdetermination theory applied to health contexts. Perspectives on Psychological Science, 7(4), 325-340. https://doi.org/10.1177/ 1745691612447309

Nguyen, T. T., Ryan, R. M., \& Deci, E. L. (2018). Solitude as an approach to affective self-regulation. Personality and Social Psychology Bulletin, 44(1), 92-106. https://doi.org/10.1177/ 0146167217733073

Nguyen, T. T., Werner, K. M., \& Soenens, B. (2019). Embracing metime: Motivation for solitude during transition to college. Motivation and Emotion, 43(4), 571-591. https://doi.org/10. 1007/s11031-019-09759-9

Pauly, T., Lay, J. C., Nater, U. M., Scott, S. B., \& Hoppmann, C. A. (2017). How we experience being alone: Age differences in affective and biological correlates of momentary solitude. Gerontology, 63(1), 55-66. https://doi.org/10.1159/000450608

Pauly, T., Lay, J. C., Scott, S. B., \& Hoppmann, C. A. (2018). Social relationship quality buffers negative affective correlates of everyday solitude in an adult lifespan and an older adult sample. Psychology and Aging, 33(5), 728-738. https://doi.org/10.1037/ pag0000278

Pavot, W., Diener, E., \& Fujita, F. (1990). Extraversion and happiness. Personality and Individual Differences, 11(12), 1299-1306. https://doi.org/10.1016/0191-8869(90)90157-m 
Pedersen, D. M. (1979). Dimensions of privacy. Perceptual and Motor Skills, 48(3_suppl), 1291-1297. https://doi.org/10.2466/pms.1979. 48.3c. 1291

Pedersen, D. M. (1997). Psychological functions of privacy. Journal of Environmental Psychology, 17(2), 147-156. https://doi.org/10. 1006/jevp.1997.0049

Pedersen, D. M. (1999). Model for types of privacy by privacy functions. Journal of Environmental Psychology, 19(4), 397-405. https://doi.org/10.1006/jevp.1999.0140

Pressman, S. D., Cohen, S., Miller, G. E., Barkin, A., Rabin, B. S., \& Treanor, J. J. (2005). Loneliness, social network size, and immune response to influenza vaccination in college freshmen. Health Psychology, 24(3), 297-306. https://doi.org/10.1037/0278-6133. 24.3.297

Reis, H. T., Sheldon, K. M., Gable, S. L., Roscoe, J., \& Ryan, R. M. (2000). Daily well-being: The role of autonomy, competence, and relatedness. Personality and Social Psychology Bulletin, 26(4), 419-435. https://doi.org/10.1177/0146167200266002

Roozen, H. G., Evans, B. E., Wiersema, H., \& Meyers, R. J. (2009). The influence of extraversion on preferences and engagement in pleasant activities in patients with substance use disorders: One size fits all? Journal of Behavior Analysis in Health, Sports, Fitness and Medicine, 2(1), 55-66. https://doi.org/10.1037/h0100374

Ryan, R. M., \& Deci, E. L. (2000). Self-determination theory and the facilitation of intrinsic motivation, social development, and wellbeing. American Psychologist, 55(1), 68-78. https://doi.org/10. 1037/0003-066x.55.1.68

Saucier, G. (1994). Mini-markers: A brief version of Goldberg's unipolar Big-Five markers. Journal of Personality Assessment, 63(3), 506-516. https://doi.org/10.1207/s15327752jpa6303_8

Scherbaum, C. A., \& Pesner, E. (2019). Power analysis for multilevel research. In S. Humphrey \& J. LeBreton (Eds.), The handbook of multilevel theory, measurement, and analysis (pp. 329-352). American Psychological Association.

Seligman, M. E. P. (2011). Flourish. Free Press.

Shaver, P., \& Buhrmester, D. (1983). Loneliness, sex-role orientation and group life: A social needs perspective. In P. B. Paulus (Ed.), Basic group processes. Springer series in social psychology (pp. 259-288). Springer. https://doi.org/10.1007/978-1-46125578-9_11

Shrout, P. E., \& Lane, S. P. (2012). Psychometrics. In M. R. Mehl \& T. S. Conner (Eds.), Handbook of research methods for studying daily life (pp. 302-320). Guilford Press.

Snijder, T. A. B. (2005). Power and sample size in multi-level designs. In B. S. Everitt \& D. C. Howell (Eds.), Encyclopedia of statistics in behavioral science (pp. 1570-1573). Wiley.

Steger, M. F., Frazier, P., Oishi, S., \& Kaler, M. (2006). The meaning in life questionnaire: Assessing the presence of and search for meaning in life. Journal of Counseling Psychology, 53(1), 80-93. https://doi.org/10.1037/0022-0167.53.1.80

Stillman, T. F., Baumeister, R. F., Lambert, N. M., Crescioni, A. W., DeWall, C. N., \& Fincham, F. D. (2009). Alone and without purpose: Life loses meaning following social exclusion. Journal of Experimental Social Psychology, 45(4), 686-694. https://doi.org/ 10.1016/j.jesp.2009.03.007

Trougakos, J. P., Hideg, I., Cheng, B. H., \& Beal, D. J. (2014). Lunch breaks unpacked: The role of autonomy as a moderator of recovery during lunch. Academy of Management Journal, 57(2), 405-421. https://doi.org/10.5465/amj.2011.1072

Tsai, J. L., Knutson, B., \& Fung, H. H. (2006). Cultural variation in affect valuation. Journal of Personality and Social Psychology, 90(2), 288-307. https://doi.org/10.1037/0022-3514.90.2.288

Vansteenkiste, M., \& Ryan, R. M. (2013). On psychological growth and vulnerability: Basic psychological need satisfaction and need frustration as a unifying principle. Journal of Psychotherapy Integration, 23(3), 263-280. https://doi.org/10.1037/a0032359

van Zyl, C. J. J., Dankaert, E., \& Guse, T. (2018). Motivation for solitude: A cross-cultural examination of adolescents from collectivist and individualist cultures in South Africa. Journal of Child and Family Studies, 27(3), 697-706. https://doi.org/10.1007/s10826017-0916-0

Wang, Y. (2006). Culture and solitude: Meaning and significance of being alone [Unpublished master's thesis]. University of Massachusetts, Amherst.

Watson, D., Clark, L. A., \& Tellegen, A. (1988). Development and validation of brief measures of positive and negative affect: The PANAS scales. Journal of Personality and Social Psychology, 54, 1063-1070. http://doi.org/10.1037/0022-3514.54.6.1063

Weinstein, N., \& Nguyen, T.-V. (2020). Motivation and preference in isolation: A test of their different influences on responses to selfisolation during the COVID-19 outbreak. Royal Society Open Science, 7(5), 200458. https://doi.org/10.1098/rsos.200458

\section{Author Biographies}

Dwight C. K. Tse is a lecturer at the School of Psychological Sciences and Health, University of Strathclyde Glasgow, G1 1QE, UK.

Jennifer C. Lay is a lecturer at the Department of Psychology, University of Exeter, Exeter, EX4 4QG, UK.

Jeanne Nakamura is an associate professor of psychology and codirector of the Quality of Life Research Center, Claremont Graduate University, Claremont, CA 91711, USA.

Handling Editor: Richard Slatcher 